31. Saccomanno MF, DE leso C, Milano G (2014) Acromioclavicular joint instability: anatomy, biomechanics and evaluation. Joints 2:87-92

32. Saier T, Venjakob AJ, Minzlaff $P$ et al (2015) Value of additional acromioclavicular cerclage for horizontal stability in complete acromioclavicular separation: a biomechanical study. Knee Surg Sports Traumatol Arthrosc 23:1498-1505

33. Schaefer FK, Schaefer PJ, Brossmann J et al (2006) Experimental and clinical evaluation of acromioclavicular joint structures with new scan orientations in MRI. Eur Radiol 16:1488-1493

34. Scheibel M, Droschel S, Gerhardt C et al (2011) Arthroscopically assisted stabilization of acute high-grade acromioclavicular joint separations. Am J Sports Med 39:1507-1516

35. Scheibel M, Ifesanya A, Pauly S et al (2008) Arthroscopically assisted coracoclavicular ligament reconstruction for chronic acromioclavicular joint instability. Arch Orthop Trauma Surg 128:1327-1333

36. Schlegel TF, Burks RT, Marcus RL et al (2001) A prospective evaluation of untreated acute grade III acromioclavicular separations. Am J Sports Med 29:699-703

37. Song T, Yan X, Ye T (2016) Comparison of the outcome of early and delayed surgical treatment of complete acromioclavicularjoint dislocation. Knee Surg Sports Traumatol Arthrosc 24:1943-1950

38. Tauber M (2013) Management of acute acromioclavicular joint dislocations: current concepts. Arch Orthop Trauma Surg 133:985-995

39. Tauber M, Gordon K, Koller H et al (2009) Semitendinosus tendon graft versus a modified Weaver-Dunn procedure for acromioclavicular joint reconstruction in chronic cases: a prospective comparative study. Am J Sports Med 37:181-190

40. Tauber M, Koller H, Hitzl W et al (2010) Dynamic radiologic evaluation of horizontal instability in acute acromioclavicular joint dislocations. Am J Sports Med 38:1188-1195

41. Tischer T, Salzmann GM, El-Azab H et al (2009) Incidence of associated injuries with acute acromioclavicular joint dislocations types III through V. Am J Sports Med 37:136-139

42. Venjakob AJ, Salzmann GM, Gabel F et al (2013) Arthroscopically assisted 2-bundle anatomic reduction of acute acromioclavicular joint separations: 58-month findings. Am J Sports Med 41:615-621

43. Zumstein MA, Schiessl P, Ambuehl B et al (2017) New quantitative radiographic parameters for vertical and horizontal instability in acromioclavicular joint dislocations. Knee Surg Sports Traumatol Arthrosc. https://doi.org/10.1007/s00167-0174579-6

\title{
Erratum
}

Orthopäde $2018 \cdot 47: 167$

https://doi.org/10.1007/s00132-017-3521-4

Online publiziert: 5. Januar 2018

(c) Springer Medizin Verlag GmbH, ein Teil von Springer Nature 2018

CrossMark

C. Chiari · S. Walzer · D. Stelzeneder - M. Schreiner $\cdot$ R. Windhager

Universitätsklinik für Orthopädie, Medizinische Universität Wien, Wien, Österreich

\section{Correction: Therapeutische Anwendung von Stammzellen in der Orthopädie}

\author{
Correction: \\ Orthopäde 2017 \\ https://doi.org/10.1007/s00132-017- \\ $3475-6$
}

Sehr geehrte Leserin, sehr geehrte Leser,

leider wurde im oben genannten Beitrag im Abbildungsverweis auf die Abb. 3 und Abb. 4 auf einen falschen Fall hingewiesen. Korrekt muss es lauten: In Abb. 3 ist ein Fallbeispiel einer 15-jährigen Patientin dargestellt, die bei einem Grad-IVKnorpeldefekt des lateralen Femurkondylus mit der beschriebenen Methode erfolgreich behandelt wurde. Abb. 4 zeigt ein Fallbeispiel einer 24-jährigen Patientin, die wegen eines osteochondralen Talusdefektes operiert wurde.

Wir bitten um Berücksichtigung der Korrektur und entschuldigen uns für den Fehler.

Die Redaktion

\section{Korrespondenzadresse}

Ao. Univ. Prof. Dr. C. Chiari, MSc

Universitätsklinik für Orthopädie, Medizinische Universität Wien

Währinger Gürtel 18-20, 1090 Wien, Österreich catharina.chiari@meduniwien.ac.at
Die Online-Version des Originalartikels ist unter https://doi.org/10.1007/s00132-017-3475-6zu finden. 